\title{
MEDIDA COMPUTADORIZADA DA EVOLUÇÃO DA ÁREA CICATRICIAL TRATADA COM ULTRASSOM TERAPÊUTICO E CITRATO DE SILDENAFILA
}

\author{
Gisele Schiochet ${ }^{1}$ \\ Laura Caon ${ }^{2}$ \\ Gabriela Maria Locatelli ${ }^{3}$ \\ Francieli Jaqueline Vieira ${ }^{4}$ \\ Caren Lis Albring 5 \\ Kaue Cesar Rossi ${ }^{6}$ \\ Otávio Ajala Fiorentin ${ }^{7}$ \\ Álvaro Vargas Junior ${ }^{8}$ \\ Nei Fronza ${ }^{9}$ \\ Débora Cristina Olsson ${ }^{10^{*}}$
}

SCHIOCHET, G.; CAON, L.; LOCATELLI, G. M.; VIEIRA, F. J.; ALBRING, C. L.; ROSSI, K. C.; FIORENTI, O. A.; VARGAS JUNIOR, A.; FRONZ, N.; OLSSON, D. C. Medida computadorizada da evolução da área cicatricial tratada com ultrassom terapêutico e citrato de sildenafila. Arq. Cienc. Saúde UNIPAR, Umuarama, v. 23, n. 3, p, 197-212, set./dez. 2019.

RESUMO: Objetivou-se comparar a evolução da cicatrização de feridas cirúrgicas com cinco protocolos de tratamento através de análise planimétrica. Grupos de 12 ratos Wistar, foram alocados em cinco estudos experimentais: A- controle negativo ; B- ferida cirúrgica, tratada com ultrassom terapêutico (UST) desligado ; C- ferida tratada somente com citrato de sildenafila ; D- ferida tratada com citrato de sildenafila e UST e grupo E-ferida tratada somente com UST. A evolução cicatricial foi acompanhada diariamente e avaliada por imagem fotográfica computadorizada aos sete, 14 e 21 dias. A aplicação do UST pulsado, com doses $1 \mathrm{MHZ}$ e $0,4 \mathrm{Wcm}^{2}$ reduziu o tempo de cicatrização epitelial em condições experimentais, favorecendo precocemente a reparação tecidual com efeitos qualitativos superiores ao tratamento com citrato de sildenafila (CS). A mensuração computacional para evolução da cicatrização de ferida dérmica mostrou-se um recurso de fácil aplicação sendo de baixo custo e eficiente para a aplicabilidade na rotina médica veterinária.

PALAVRAS-CHAVE: Cicatrização. Derme. Fonoforese. Fosfodiesterase tipo 5.

\section{COMPUTERIZED MEASUREMENT OF THE EVOLUTION OF CICATRICIAL AREA TREATED WITH ULTRASOUND AND SILDENAFIL CITRATE}

\begin{abstract}
This study aimed at comparing the evolution of healing of surgical wounds with five treatment protocols through planimetric measurement. Groups of 12 Wistar rats were allocated in five experimental studies: A - negative control; B - surgical wound treated with therapeutic ultrasound turned off; $\mathrm{C}$ - surgical wound treated with sildenafil citrate; D - wound treated with sildenafil citrate and therapeutic ultrasound; and group E - wound treated only with therapeutic ultrasound. The healing progress was monitored daily and assessed by computed photographic image at seven, 14 and 21 days. It was concluded that the application of pulsated therapeutic ultrasound on surgical wounds at $1 \mathrm{MHz}$ and $0.4 \mathrm{Wcm}^{2}$ doses reduces the epithelial healing time in experimental conditions, favoring the early repair of tissue with qualitative effects superior than the ones found in the treatment with sildenafil citrate (SC). The computational measurement for the evolution of the dermal wound healing proved to be an easy-to-apply resource, with a low cost and great efficiency for the applicability in the veterinary medical routine.
\end{abstract}

KEYWORDS: Healing. Dermis. Phosphodiesterase type 5. Phonophoresis.

Introdução

A maioria dos estudos desenvolvidos na área de reparação tecidual tem como objetivo acelerar o mecanismo regenerativo, restabelecer a função fisiológica da região afetada, porém, a ausência de elementos como o colágeno, pode interferir no resultado final da cicatrização (OLSSON et al., 2006). A reparação de ferimentos inicia após uma lesão e sua

DOI: 10.25110 /arqsaude.v23i3.2019.6822

${ }^{1}$ Discente de Medicina Veterinária Instituto Federal Catarinense - Campus Concórdia, Rodovia SC 283-Km 17-CEP 89703-720. Concórdia, SC, Brasil. E-mail: giseleschiochet@gmail.com.

${ }^{2}$ Discente de Medicina Veterinária Instituto Federal Catarinense - Campus Concórdia, Rodovia SC 283-Km 17-CEP 89703-720. Concórdia, SC, Brasil. E-mail: laura.caon@hotmail.com.

${ }^{3}$ Discente de Medicina Veterinária. Instituto Federal Catarinense - Campus Concórdia, Rodovia SC 283-Km 17-CEP 89703-720. Concórdia, SC, Brasil. E-mail: hbi_locatelli@hotmail.com.

${ }^{4}$ Discente de Medicina Veterinária. Instituto Federal Catarinense - Campus Concórdia, Rodovia SC 283-Km 17-CEP 89703-720. Concórdia, SC, Brasil. E-mail: francieli premier@hotmail.com.

${ }_{5}^{5}$ Discente de Medicina Veterinária. Instituto Federal Catarinense - Campus Concórdia, Rodovia SC 283-Km 17-CEP 89703-720. Concórdia, SC, Brasil. E-mail caren.lis@hotmail.com.

${ }^{6}$ Discente de Medicina Veterinária. Instituto Federal Catarinense - Campus Concórdia, Rodovia SC 283-Km 17-CEP 89703-720. Concórdia, SC, Brasil. E-mail: kaue_rossi@hotmail.com

${ }^{7}$ Engenheiro Agrônomo. Universidade do Estado de Santa Catarina-UDESC-CAV. Avenida Luiz de Camões 1370 . Coral. CEP 88523000 - Lages, SC Brasil. E-mail:otavioaf@hotmail.com

${ }^{8}$ Bioquímico, Docente do Curso de Medicina Veterinária. Instituto Federal Catarinense - Campus Concórdia, Rodovia SC 283-Km 17-CEP 89703-720. Concórdia, SC, Brasil. E-mail: alvaro.vargas@ifc.edu.br

${ }^{9}$ Bioquímico, Docente do Curso de Medicina Veterinária. Instituto Federal Catarinense - Campus Concórdia, Rodovia SC 283-Km 17-CEP 89703-720. Concórdia, SC, Brasil. E-mail: nei.fronza@ifc.edu.br

${ }^{10}$ Médica Veterinária. Docente do Curso de Medicina Veterinária. Instituto Federal Catarinense - Campus Concórdia, Rodovia SC 283-Km 17-CEP 89703720. Concórdia, SC, Brasil. E-mail: debora.olsson@ifc.edu.br. Autor para correspondência. 
interferência corresponde a uma combinação de eventos incluindo deiscência da ferida, cicatrização retardada, contraturas, infecções, insuficiência arterial e congestão venosa que desempenham papéis importantes (HEDLUNG, 2007; FARSAEI et al., 2015), como a perfusão insuficiente que pode causar uma ferida crônica ou necrose, dependendo o grau de isquemia (ARSLANTAS; ARSLANTAS, 2015).

Com base nestes mecanismos, modalidades físicas como ondas mecânicas e farmacológicas como, simpatolíticos, vasodilatadores, inibidores da prostaglandina, e liberadores de radicais livres têm sido estudados como potencial de melhoria na sobrevida dos tecidos, porém, com variações nos graus de sucesso (PELIZZARI et al., 2012; FARSAEI et al., 2015).

Recentemente, pesquisas experimentais têm sido focadas em diferentes efeitos do citrato de sildenafila (CS) um inibidor da fosfodiesterase conhecido por seus efeitos na disfunção erétil e no processo de cicatrização de ferida (ARSLANTAS; ARSLANTAS, 2015). Esse processo inclui o óxido nítrico (NO) que contribui para a vasodilatação e neoangiogênese, além de auxiliar na regulação, proliferação celular, síntese de colágeno e epitelização (FARSAEI et al., 2015). Como o CS aumenta a liberação de NO, está sendo usado para o reparo de lesões (GÜRSOY et al., 2014).

Além dos recursos químicos, a possibilidade de estimular o fechamento de lesões por meio de recursos físicos, como as ondas mecânicas, tem sido objetivo de investigação de inúmeros pesquisadores (OLSSON et al., 2006; BATKIR et al., 2018). Por esse motivo e diante de questionamentos sobre o tratamento de feridas com meios físicos e químicos, este estudo se propôs acompanhar a evolução cicatricial de ferida iatrogênica e analisar macroscopicamente, por meio de planimetria para mensuração da área da lesão, os efeitos de ondas mecânicas do ultrassom terapêutico (UST) pulsado, em modalidade fonoforese com CS como adjuvante na cicatrização epitelial.

\section{Material e Métodos}

Este estudo foi aprovado pelo Comitê em Ética de Uso de Animais (CEUA) do Instituto Federal Catarinense Campus Concórdia, SC, protocolo n ${ }^{\mathrm{o}}$ 30/2015.

Utilizaram-se ratos Wistar albinos (Rattus norvegicus), machos, provenientes do biotério central da Universidade Federal de Santa Catarina (UFSC), quatro meses de idade, pesando 400 a $500 \mathrm{~g}$. Os animais foram alojados em gaiolas, de acordo com CONCEA (Resolução normativa $n^{\circ}$ 15 de 16/12/2013), durante quinze dias, para aclimatização e adaptação ambiental e humana. A temperatura e a umidade relativa do ar foram controladas entre $22^{\circ} \mathrm{C}\left( \pm 2^{\circ} \mathrm{C}\right)$ e $55 \%$ $( \pm 5 \%)$ respectivamente. Disponibilizou-se ração comercial peletizada específica para a espécie e água ad libitum (KILKENNY et al., 2010).

Alocaram-se os animais em cinco grupos experimentais (A, B, C, D e E) com 12 ratos. Os animais do grupo $\mathrm{A}$ foram submetidos à indução de ferida cirúrgica e não receberam tratamento; $\mathrm{O}$ grupo $\mathrm{B}$ foi submetido à indução de ferida seguido de gel acoplamento hidrossolúvel e tratado com UST desligado, por cinco minutos; Nos animais do grupo $\mathrm{C}$ foi realizada indução de ferida e receberam tratamento tópico com 10mg de citrato de sildenafila (CS) (BAS-
SON et al., 2002; DERICI et al., 2010; PAAUW et al, 2017; KULSHRESTHA et al., 2019) sobre a lesão; No grupo D após indução cirúrgica, foi utilizado o tratamento tópico com 10mg de CS seguido de UST pulsado, por cinco minutos; Os animais do grupo E receberam tratamento somente com UST com gel acoplamento hidrossolúvel por cinco minutos. Todos os animais foram redistribuídos em três subgrupos de quatro animais de acordo com o tempo de avaliação planimétrica aos sete, 14 e 21 dias.

Para a indução anestésica, os animais foram submetidos à anestesia geral inalatória, com isoflurano, vaporizado em oxigênio a $100 \%$, ao efeito, administrado por meio de máscara facial. Foi feita uma incisão de $1,5 \mathrm{~cm}^{2}$ na região dorsal caudal da borda escapular, demarcada com molde vazado e lápis dérmico precedido por tricotomia e receberam analgesia com cloridrato de tramadol $(5 \mathrm{mg} / \mathrm{kg})$, por via intraperitoneal no pós-operatório imediato. Nos grupos que receberam CS, o gel permaneceu por cinco minutos sobre a ferida. A frequência do UST utilizada foi protocolada em $1 \mathrm{MHz}$, ERA $3,5 \mathrm{~cm}^{2}$, modo pulsado, com intensidade de $0,4 \mathrm{Wcm}^{2}$ em movimentos circulatórios contínuos (BYL et al., 1992; OLSSON et al., 2006; PELLIZARI, et al., 2012).

A análise da evolução cicatricial foi realizada com base na avaliação fotográfica planimétrica da área da lesão. Sob o método manual de contenção, os animais foram posicionados ventro-dorsalmente e com uma câmera digital (SONY NEX-C3®), fixada em $15 \mathrm{~cm}$ de altura por um tripé, obtiveram-se os registros fotográficos utilizando ao lado da ferida uma régua para delimitar o tamanho da lesão $\left(\mathrm{em} \mathrm{cm}^{2}\right)$. Foram fotografadas três imagens da ferida de cada animal; a primeira, não se posicionou a régua, na segunda imagem a régua foi posicionada horizontalmente ao lado da ferida e, na última imagem, posicionou-se a régua verticalmente ao lado da ferida. A evolução das feridas foi fotografada diariamente para demonstrar a aparência do seu tamanho de forma qualitativa (FALCÃO et al., 2001; FERNANDES et al., 2015).

As imagens foram transferidas para um computador e com a ajuda do Software GIMP2® gratuito (FERNANDES et al., 2015), onde mensurou e calculou-se a área e o processo de cicatrização diária de todos os grupos. Após a cicatrização total das feridas realizou-se uma média entre os grupos fixados na evolução de reparo, relatando assim a efetividade macroscópica entre os tratamentos no processo dos diferentes protocolos.

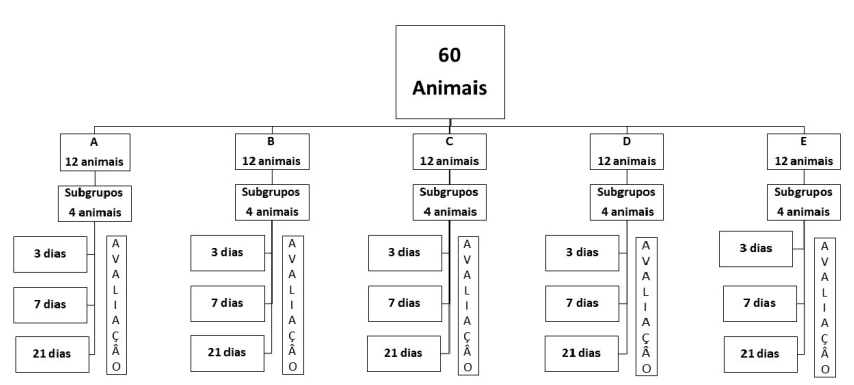

Figura 1: Fluxograma dos cinco grupos experimentais submetidos a tratamento. A-grupo indução de ferida cirúrgica sem tratamento; B-grupo indução de ferida tratado com UST desligado; C-grupo ferida tratada com 10mg de CS; D-grupo ferida tratada com $10 \mathrm{mg}$ de CS e UST pulsado; E-grupo ferida tratada com UST pulsado. 
As variáveis medidas foram testadas pela análise de variância (ANOVA). O modelo experimental foi o de blocos inteiramente casualizados. Os contrastes de médias foram avaliados pelo teste de Scheffé a $5 \%(\leq 0,05)$ de probabilidade e para a confecção dos gráficos utilizou-se o teste " $t$ " de Student $(\leq 0,05)$.

\section{Resultados e Discussão}

O fechamento de uma lesão cutânea se dá por contração da ferida, entretanto, não ocorre disfunção do tecido nem perda de atividade nos tecidos de animais, já em humanos, uma contração demasiadamente pequena retarda o fechamento com consequente deformidade e disfunção (FERREIRA et al., 2008). Na avaliação diária das feridas, neste trabalho, não foi observado retardamento ou deformidade tecidual assim como, sinais de infecção ou sangramento que pudessem interferir no mecanismo cicatricial.

Embora universalmente não haja uma metodologia para determinar o tamanho de uma ferida, a escolha pelo método de software computacional demonstra precisão na leitura qualitativa da extensão das lesões, principalmente porque ele considera o contorno irregular da lesão nos estágios avançados do processo cicatricial, desde grandes a pequenas feridas (FERNANDES et al., 2015), como foi o caso deste experimento com feridas iatrogênicas de $1,5 \mathrm{~cm}^{2}$. Porém, para Falcão et al. (2001) a escolha de planimetria com a utilização de papel gráfico constitui um processo alternativo e de precisão equivalente ao computacional e, para Medrano (2011), pode-se usar ainda, como auxílio, um paquímetro tendo um relógio como uma guia de medição. A escolha computacional para esta pesquisa ocorreu devido à confiabilidade precisa da demarcação, redução de margem de erro, facilidade de manipulação do programa e fácil acesso gratuito de seu software nas redes sociais.

Apesar de o método computadorizado ser confiável, Farsaei (2015), preconiza a necessidade de dois avaliadores treinados para as percepções visuais. Neste estudo utilizou-se um avaliador para a captação e, de um avaliador para a determinação das marcações antecipando as imagens. Entretanto, Boyce; Logan (1994) e Abu et al (2018) afirmam que qualquer método utilizado requer examinadores bem treinados, mas sempre haverá intra e intervariabilidade dos avaliadores, portanto, o uso dos mesmos pesquisadores durante todo o estudo torna fundamentada a abordagem e a metodologia mais confiável.

Nos resultados, as taxas médias de redução da área $\left(\mathrm{cm}^{2}\right)$ da lesão cutânea nos animais foram: -0,073, -0,087, $-0,069,-0,124$ e $-0,123$ para os tratamentos A, B, C, D e E, respectivamente (Tabela 1, Figura 2). Os tratamentos A, B e $\mathrm{C}$ apresentaram as menores taxas de redução da área $\left(\mathrm{cm}^{2}\right)$ da lesão e consequentemente maior tempo para cicatrização total (Tabela 1, Figura 2 e 3), até os 14 dias.

O grupo A e B, ao longo dos 18 e 16 dias respectivamente, desenvolveram um tecido cicatricial prematuro ao tempo previsto de avaliação final que seria de 21 dias (Figura 3). A área média inicial da lesão, imediatamente depois de produzida no grupo A foi de $2,1 \mathrm{~cm}^{2}$, diminuindo para $0,46 \mathrm{~cm}^{2}$ no sétimo dia; $0,07 \mathrm{~cm}^{2}$ no décimo quarto dia e $0,0008 \mathrm{~cm}^{2}$ no décimo oitavo dia de cicatrização (Tabela 1 , Figura 3).
Tabela 1: Médias e desvios padrão da área $\left(\mathrm{cm}^{2}\right)$ da lesão cutânea cirúrgica de ratos albinos Wistar (Rattus norvegicus) submetidos a diferentes protocolos de tratamentos $\mathrm{A}^{*}, \mathrm{~B}^{* *}$, $\mathrm{C}^{* *}, \mathrm{D}^{* *}$ e $\mathrm{E}^{*}$.

\begin{tabular}{l|c|c|c}
\hline & DIA 7 & DIA 14 & DIA 21 \\
\hline GRUPO A & $0,46 \pm(0,05)$ & $0,07 \pm(0,02)$ & $0,00 \pm(0,00)$ \\
\hline GRUPO B & $0,49 \pm(0,01)$ & $0,03 \pm(0,02)$ & $0,00 \pm(0,00)$ \\
\hline GRUPO C & $0,45 \pm(0,06)$ & $0,00 \pm(0,00)$ & $0,00 \pm(0,00)$ \\
\hline GRUPO D & $0,78 \pm(013)$ & $0,02 \pm(0,01)$ & $0,00 \pm(0,00)$ \\
\hline GRUPO E & $0,86 \pm(0,03)$ & $0,07 \pm(0,01)$ & $0,00 \pm(0,00)$ \\
\hline
\end{tabular}

**Grupos Tratamentos

No grupo B, a área média da lesão foi de $1,41 \mathrm{~cm}^{2}$ de imediato e de $0,49 \mathrm{~cm}^{2}$ no sétimo dia, finalizando com $0,007 \mathrm{~cm}^{2}$ no dia 16 . Assim como no grupo $\mathrm{C}$, que recebeu somente CS topicamente pelo tempo total de 21 dias, a área média aos sete dias foi de $0,45 \mathrm{~cm}^{2}$, aos quatorze dias $0,0 \mathrm{~cm}^{2}$ demostrando que no décimo quarto dia de observação a cicatrização apresentou-se completa (Figura. 3), entretanto, sua média da taxa de redução da área apresentou-se maior que as dos grupos D e E, apesar de o tratamento ter sido eficiente (Tabela 1, Figura 3).

Os tratamentos dos grupos D e E apresentaram as maiores taxas de redução da área $\left(\mathrm{cm}^{2}\right)$ da lesão, implicando em menor tempo até a cicatrização total da ferida cirúrgica (Figura 2, Tabela 1). O tempo total de cicatrização entre os grupos foi de 14 e 15 dias respectivamente (Figura 2 e 3 ). No grupo em que os animais receberam tratamento com $10 \mathrm{mg}$ de CS para fonoforese, a área média total da lesão foi de $1,88 \mathrm{~cm}^{2}$, de $0,78 \mathrm{~cm}^{2}$ no sétimo dia, e de e $0,02 \mathrm{~cm}^{2}$ no décimo quarto dia, atingindo uma cicatrização completa antecipadamente aos 21 dias (Figura 3).

Para o tratamento E (GU) as avaliações foram de $2,2 \mathrm{~cm}^{2}$ na sua área total; $0,86 \mathrm{~cm} 2$ no sétimo dia e $0,07 \mathrm{~cm} 2$ no décimo quarto dia, desta forma, apresentando o menor tempo de evolução cicatricial, diferindo estatisticamente $(p \leq 0,05)$ daqueles valores correspondentes, no experimento controle, tratados somente com gel acoplamento hidrossolúvel e somente com CS (Tabela 1).

Comparando todos os grupos, observou-se que cicatrizaram antes de 21 dias de tratamento. Este fato pode ser atribuído ao tamanho da ferida, mas, apesar disso, os grupos que receberam os tratamento C, D e E apresentaram uma melhor resposta cicatricial que o grupo controle A e B. O grupo C, apesar de ter completado sua cicatrização em 14 dias, sua área de mensuração foi estatisticamente maior quando comparados aos grupos D e E.

De acordo com Derici et al. (2010) em estudo do efeito de CS em feridas, revelaram que aos quatro, 14 e 21 dias a proporção volumétrica de células inflamatórias foi menor nos grupos tratados que o controle e como resultado obtiveram precocidade de cicatrização e uma melhor formação de tecido de granulação aos cinco, sete e 10 dias e, maior neovascularização aos 14 dias. Resultado semelhante foi observado neste experimento onde se percebeu macroscopicamente um tecido com maior vitalidade e formação de granulação sem apresentar visualmente aparência de necrose com completa cicatrização aos 14 dias (Figura 3). 
O fármaco citrato de sildenafila (CS) pode representar uma terapia de resgate em pacientes que não respondem a terapia epitelial convencional. Alternativamente, ele também pode ser a primeira escolha em pacientes nos quais outros medicamentos são contraindicados ou de difícil acesso, concordando com Gürsoy et al. (2014) e Farsaei et al. (2015) que referem ser o CS um novo agente para tratamento de feridas abertas devido sua facilidade de administração e efeitos colaterais aceitáveis.

No tratamento D e E, foram usados como tratamento tópico adjuvante e carreador da ação medicinal pelas ondas mecânicas da terapia ultrassônica. Os dois tratamentos destacaram-se significativamente $(\mathrm{p} \leq 0,05)$ quando comparados aos outros grupos por apresentarem uma menor área de mensuração no qual revela melhor qualidade da cicatrização macroscópica. A rápida capacidade cicatricial foi verificada pela expressiva redução de tamanho que pode ter ocorrido sugestivamente por ativação da fibroplasia, presente na fase de proliferação celular incluindo formação de tecido de granulação, diminuição de células inflamatórias e componentes neovasculares (OLSSON et al., 2006; GARBUIO et al., 2018). As características de evolução rápida foram verificadas de forma macroscópica e através do estudo planimétrico precocemente nesses grupos quando da aplicação do UST pulsado.
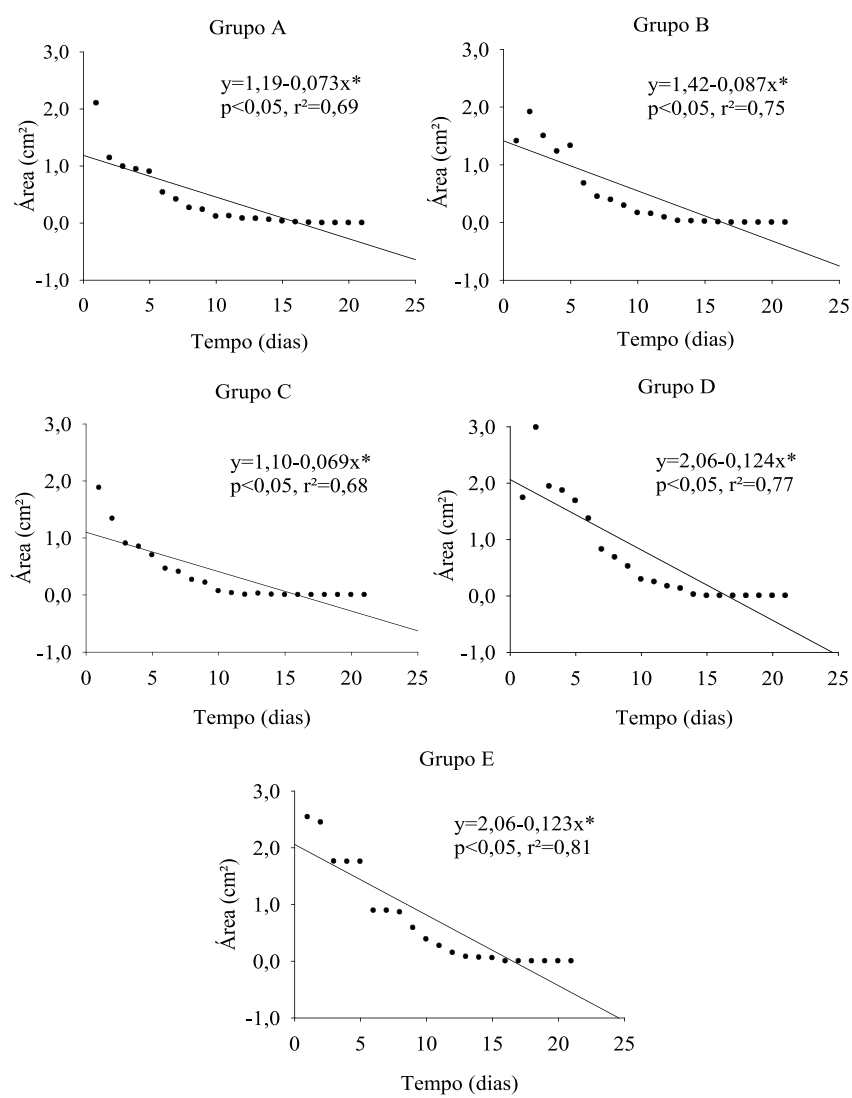

Figura 2: Redução da área $\left(\mathrm{cm}^{2}\right)$ da lesão cutânea cirúrgica de ratos albinos Wistar (Rattus norvegicus) submetidos a diferentes tratamentos. A reta representa a regressão linear ajustada aos dados, sendo, $\mathrm{y}=$ área da lesão, $\mathrm{x}=$ tempo em dias.

*Equações significativas pelo teste " $t$ ", de Student $(p<0,05)$. A-ferida cirúrgica sem tratamento; B-ferida tratado com UST desligado; C- ferida tratada com $10 \mathrm{mg}$ de CS; D-ferida tratada com 10mg de CS e UST pulsado; E-ferida tratada com UST pulsado.

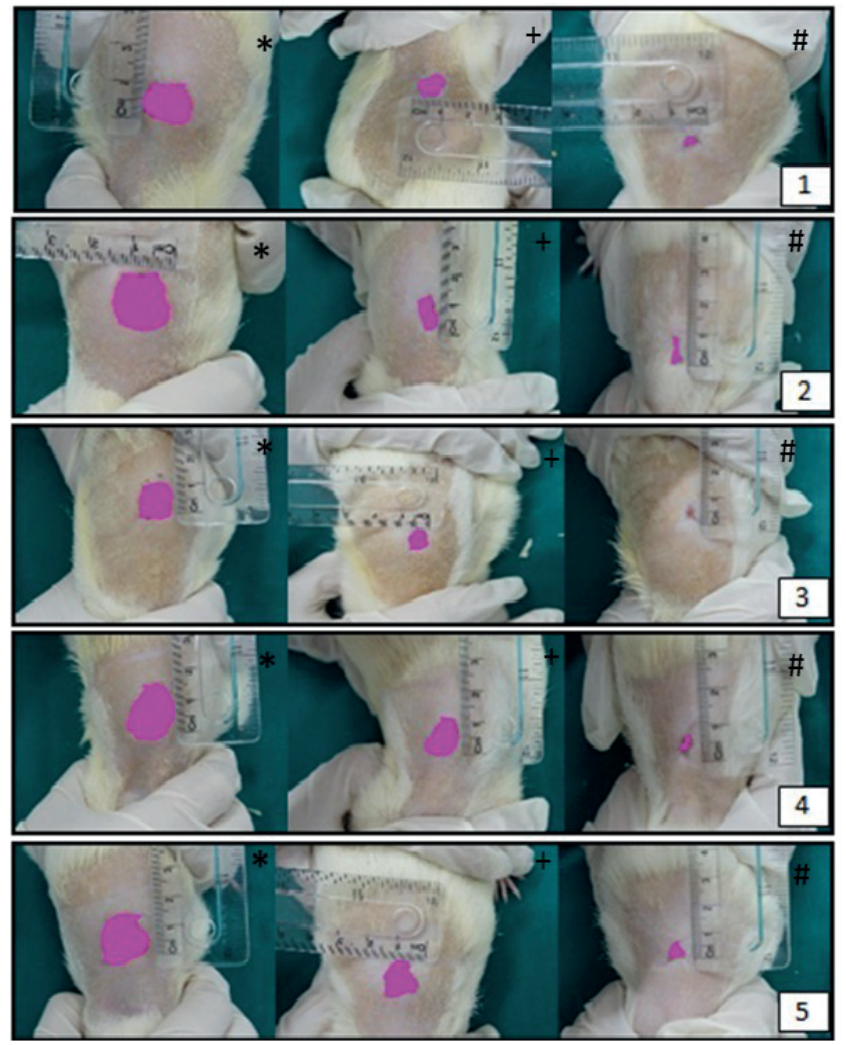

Figura 3: Evolução da cicatrização tecidual da lesão cutânea cirúrgica de ratos albinos Wistar (Rattus norvegicus) submetidos a grupos de tratamentos, avaliados por planimetria de acordo com a área $\left(\mathrm{cm}^{2}\right)$ e demarcadas pelo software GIMP2 ao zero* ${ }^{*}$ sete $^{+}$e $14^{\#}$ dias, respectivamente.

Legenda: Imagem 1 - Grupo A; Imagem 2 - Grupo B; Imagem 3 - Grupo C; Imagem 4 - Grupo D; Imagem 5 - Grupo E. Software GIMP2® .

Os protocolos usados neste experimento produziram efeitos tissulares positivos, não sendo observadas alterações nas feridas, entretanto, Fernandes et al. (2003) descrevem que dependendo da intensidade, tempo e frequência das aplicações, podem haver retardo na cicatrização e ainda, referem que quando utilizados o UST numa intensidade menor ocorre maior ativação de fibroblastos, hidroxiprolina e colágeno.

Os grupos D e E utilizaram UST, porém, no grupo $\mathrm{D}$ os resultados sugerem que a fonoforese pode ter causado aumento da permeabilidade celular, incentivando a penetração do CS na pele e essa absorção pode ser influenciada pelo modo de onda pulsada (MILLER, 2000; PELIZZARI et al., 2012; TAKLA; REZK-ALLAH, 2018). Fang et al. (1999) e Silveira et al.(2015) demonstraram em seu experimento que o UST pulsado é mais efetivo na acentuação da permeabilidade, na indução da diminuição de estresse oxidativo e resposta inflamatória do que o modo contínuo, isso contradiz os relatos descritos por Ciccone (1990) e Kozanoglu et al. (2003) que verificam o predomínio do modo contínuo dos estudos analisados.

No entanto, apesar de significativo, o grupo E, onde o tratamento utilizou somente aplicação de UST pulsado (Figura 2) apresentou dentre eles, a menor área de mensuração da ferida e maior rapidez na cicatrização das lesões. Esses achados estão de acordo com Byl et al. (1992) e Batkir et al (2018) que afirmam que a precocidade de resolução cicatri- 
cial em estudos que utilizam UST pulsado está na diminuição do processo inflamatório, aumento na força de contração e taxa de cicatrização do grupo tratado e isso se deve provavelmente a dosimetria escolhida.

$\mathrm{O}$ uso do UST, com frequência de $1 \mathrm{MHz}$, intensidade de $0,4 \mathrm{Wcm}^{2}$ por cinco minutos nos tratamentos $\mathrm{D}$ e $\mathrm{E}$, com ou sem o uso de CS se mostrou mais eficiente em acelerar o processo de cicatrização de lesões cutâneas (Figura 2 e 3). Estes achados estão de acordo com o estudo realizado por Olsson et al. (2006) ao verificarem que em feridas cirúrgicas produzidas na parede abdominal a aplicação do UST no modo pulsado, com doses da frequência de $1 \mathrm{MHz}$ e intensidade de $1 \mathrm{Wcm}^{2}$ reduz o tempo de reação inflamatória e acelera a cicatrização, favorecendo precocemente a reparação tecidual.

Farcic et al (2018) estudaram sobre os efeitos do UST pulsado no tendão de cães e ratos respectivamente, utilizando uma intensidade de $0,5 \mathrm{Wcm}^{2}$ e concluíram que nos tendões tratados ocorreu maior formação de fibras, organização de colágeno e aceleração do reparo tecidual. Ainda na espécie canina, Souza (2008) observou os efeitos do UST pulsado com intensidade de $30 \mathrm{mWcm}^{-2}$ como adjuvante na consolidação óssea, sugerindo que este tipo de tratamento pode auxiliar no reparo de fraturas de ossos longos. Outros autores também confirmam a aceleração do processo de cicatrização tecidual através do uso do UST, como é o caso de Freitas et al. (2006) e Filho et al., (2018) que afirmaram ser a terapia por UST uma forma mecânica que influência a atividade das células, plaquetas, mastócitos, macrófagos e neutrófilos envolvidos na fase inflamatória do processo de regeneração tecidual, acelerando o processo de cicatrização, reduzindo o dano oxidativo durante o reparo.

\section{Conclusão}

Os resultados obtidos permitem concluir que em feridas cirúrgicas produzidas na pele, a aplicação do ultrassom no modo pulsado, com doses na frequência de $1 \mathrm{MHz}$ e intensidade de $0,4 \mathrm{Wcm}^{2}$ reduz o tempo de cicatrização mediante de análise planimétrica, favorecendo precocemente a reparação tecidual, sendo seus efeitos qualitativos superiores ao tratamento com citrato de sildenafila. A análise computacional mostrou-se eficiente para a aplicabilidade na rotina veterinária.

\section{Agradecimento}

Ao apoio financeiro institucional PIBIC.

\section{Referências}

ABU, E. K. et al. Comparing different methods of measuring accommodative amplitude with Hofstetter $>$ s normative values in a Ghanaian population. Curr Eye Res, v. 43, n. 9, p. 1145-1150, 2018.

ARSLANTAS, R.; ARSLANTAS, M. K. Adverse Effect of Sildenafil on Healing Ischemic Wounds: Results of an In vivo Study. Ostomy Wound Manage v. 61, n. 9, p. 32-37, 2015.

BASSON, R. et al. Efficacy and safety of sildenafil citrate in women with sexual dysfunction associated with female sexual arousal disorder. J Womens Health Gend Based Med. v. 11, n. 4, p. 36777, 2002.

BATKIR, S. et al. The short-term effectiveness of low-level laser, phonophoresis, and iontophoresis in patients with lateral epicondylosis. J of Hand Ther, 2018 Feb 13. pii: S0894-1130(17)30232-6. Doi: 10.1016/j.jht.2018.01.002. [Epub ahead of print].

BOYCE, E. N.; LOGAN, E. I. Oral health assesment in dogs: study design and results. J Vet Dent, n. 11, v. 2, p. 64-70, 1994.

BYL, N. N. et al. The efeccts of phonophoresis with corticosteroids: a controlled pilot study. J Orthop Sport Physic Ther, v. 18, n. 5, pp. 590-600. 1992.

CICCONE, C. D. Pharmacology in Rehabilitation. 4. ed. F. A. Davis Company, 1990. 682p.

CONCEA. Conselho Nacional de Controle de Experimentação Animal. (Resolução normativa $n^{\circ} 15$ de 16/12/2013). Disponível em http://mctic.gov.br/mctic/ opencms/institucional/concea/paginas/legislacao.html. Acesso em: 30 mai. 2018.

DERICI, H. et al. Effect of sildenafil on wound healing: an experimental study. Lang Arch Surg. n. 395, v. 6, p. 713 718, 2010.

FALCÃO, C. S. et al. Processo modificado de reprodução e amplificação de imagem para mensuração de área por planimetria. Aplicação em feridas planas produzidas em cães, tratadas por curativos oclusivos de pele de rã. Braz J Vet Res Anim Sc. 2001

FANG, J. et al. Effect of low frequency ultrasound on the vitro percutaneous absorption of clobetasol 17- propionate. Int J Pharma, v., 191, n. 1, pp. 33-42. 1999.

FARCIC, T. S. et al. Collagen Fibers in the Healing Process of Rat Achilles Tendon Rupture Using Different Times of Ultrasound Therapy. Adv Wound Care (New Rochelle), v. 7, n. 4, p. 114-120, 2018.

FARSAEI, S. et al. Sildenafil in the treatment of pressure ulcer: a randomised clinical trial. Int Wound Jour. 2015.

FERREIRA, A. S. et al. Mensuração de área de cicatrização por planimetria após aplicação do ultra-som de baixa intensidade em pele de rato. Rev Bras Fisioter. v. 12, n. 5, p. $351-358,2008$.

FERNANDES, M. A. L. et al. Efeito do ultra-som terapêutico em tendinite experimental de equinos: estudo clínico, ultrassonográfico e histopatológico de dois protocolos. Arq Bras Med Vet Zootec, v. 55, n. 1, p. 27-34, 2003.

FERNANDES, M. A. L.; ALVES, G. E. S.; SOUZA, J. C. A. Comparing open wound measuring methods popularly used 
in experimental studies. Braz J Vet Res Animal Sci. v. 52, n. 2, p. 106-111, 2015.

FREITAS, L. S. et al. Effect of therapeutic pulsed ultrasound on parameters of oxidative stress in skeletal muscle after injury. Cell Biol Int. v. 31, n. 5, p. 482-486, 2006.

FILHO, L. F. S. et al. Effect of pulsed therapeutic ultrasound and diosmin on skeletal muscle oxidative parameters. Ultras Med Biol, v. 44, n. 2, p. 359-367, 2018.

GARBUIO, D. C. et al. Instrumentos para avaliação da cicatrização de lesões de pele: revisão integrativa. Rev. Eletr. Enf. [Internet]. 2018. Disponível em: https://doi. org/10.5216/ree.v20.49425. Acesso em: 11 maio 2019.

GÜRSOY, K. et al. Effect of topically applied sildenafil citrate on wound healing: experimental study. Bos J Basic Med Sci. v. 14, n. 3. p. 125-131, 2014.

HEDLUG, C. S. Surgery of the Integumentary System. In: FOSSUM, T. W. Small Anim Surg. 3. ed. Missouri: Mosby Elsevier, 2007. Cap. 15, p. 161-259.

KILKENNY, C. et al. Improving Bioscience Research Reporting: The ARRIVE Guidelines for Reporting Animal Research. PLoS Biol, v. 8, n. 6, e1000412, 2010.

KOZANOGLU, E. et al. Short term efficacy of ibuprofen phonophoresis versus continuous ultrasound therapy in knee osteoarthritis. Swiss Med Wkly. v. 133, n. 23-24, p. 333-338, 2003.

KULSHRESTHA, S. et al. Efficacy and dermal toxicity analysis of Sildenafil citrate based topical hydrogel formulation against traumatic wounds. Biomedicine $\&$ Pharmacotherapy, v. 112, 2019.

MEDRANO, R. C. J. Medida de la cicatrización en úlceras por presión. ¿Con qué contamos? Rincón Cien Com. v. 22, n. 1, p. 35-42, 2011.

MILLER, M. W. Gene transfection and drug delivery. Ultras Med Biol. v. 26, n. 1, p. 59-62. 2000.

OLSSON, D. C. et al. Estimulação ultra-sônica pulsada e contínua no processo cicatricial de ratos submetidos à celiotomia. Cienc Rural, v. 36, n. 3, p. 865-872, 2006.

PAAUW, N. D. et al. Sildenafil during pregnancy a preclinical meta-analysis on fetal growth and maternal blood pressure. Hyper. Aha journals, p. 1-25, 2017.

PELIZZARI, C. et al. Concentração plasmática de diclofenaco sódico em cães, submetidos à fonoforese. Arq Bras Med Vet Zootec.v. 64, n. 6, p. 1449-1455, 2012.

SAINI, N. S. et al. A preliminary study on the effect of ultrasound therapy on the healing of surgically severed Achilles tendons in five dogs. J Vet Med, v. 49, p. 321-328, 2002.
SILVEIRA, P. C. et al. Effects of phonophoresis with gold nanoparticles on oxidative stress parameters in a traumatic muscle injury model. Drug Deliv. v. 23, n. 3, p. 926-32, 2016

SOUZA, V. L. Ultra-som pulsado de baixa intensidade em fraturas diafisárias: aplicação clínica em cães. Ciênc Rural, v. 38, n. 4, 2008.

TAKLA, M. K. N.; REZK-ALLAH, S. S. Immediate effects of simultaneous application of transcutaneous electrical nerve stimulation and ultrasound phonophoresis on Active Myofascial Trigger Points: A Randomized Controlled Trial. Am J Phys Med Rehab. v. 97, n. 5, p. 332-338, 2018.

Recebido em: 03/06/2018 Aceito em: 10/06/2019 